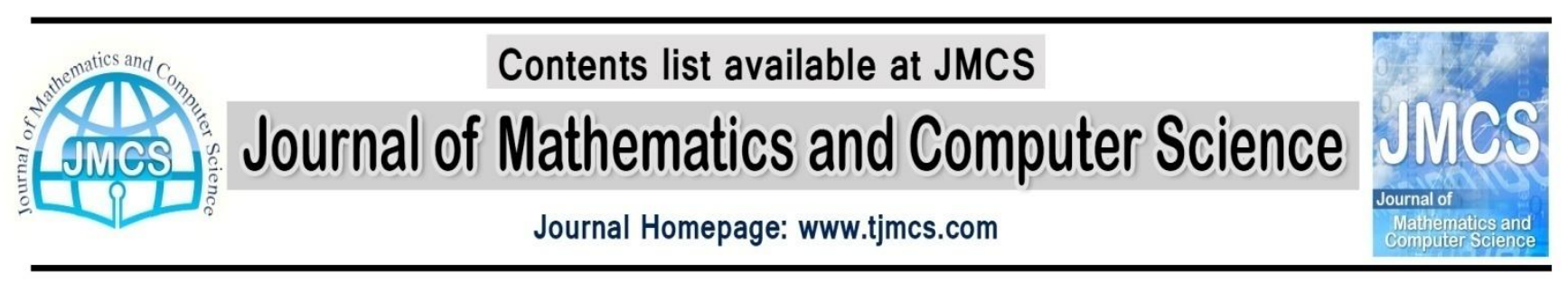

\title{
Adomian Decomposition Method for Solving Fractional Bratu-type Equations
}

\author{
Bahman Ghazanfari \\ Amaneh Sepahvandzadeh \\ Department of Mathematics, Lorestan University, \\ Khorramabad, 68137-17133, Iran \\ bahman_ghazanfari@yahoo.com \\ sepahvandzade_amane@yahoo.com
}

Article history:

Received June 2013

Accepted July 2013

Available online July 2013

\section{Abstract}

The Adomian decomposition method is proposed to solve fractional Bratu-type equations. The iteration procedure is based on a fractional Taylor series. Three examples are illustrated to show the presented method's efficiency and convenience.

Keywords: Fractional Bratu-type equation, Adomian decomposition method, Jumarie's derivative.

\section{Introduction}

Fractional differential equations are studied in various fields of physics and engineering, namely in signal processing, control engineering [1, 2], electromagnetism [3], biosciences [4], fluid mechanics [5], electrochemistry [6], diffusion processes [7], dynamic of viscoelastic material [8], continuum and statistical mechanics [9] and propagation of spherical flames [10].

In general, most of the fractional differential equations do not have exact solutions. Particularly, there is no known method for solving fractional boundary value problems exactly. Therefore several methods for the approximate solutions to classical differential equations [11] are extended to solve differential equations of fractional order numerically. These methods include, Adomian decomposition method [12], homotopy perturbation method [13-16], homotopy analysis method [17], variational iteration method [18], generalized differential transform method [19], finite difference method [20] and etc [21, 22].

In this paper, Adomian decomposition method $[23,24]$ is extended for solving fractional Bratu's initial value problem as follows:

$$
\begin{gathered}
D_{x}^{2 \alpha} u(x)+\lambda e^{u(x)}=0, \quad 0<\alpha \leq 1, \quad 0<x<1, \\
u(0)=u^{(\alpha)}(0)=0, \quad \lambda \text { is a constant, }
\end{gathered}
$$

where $\alpha$ is an order of Jumarie's fractional derivative and $D^{2 \alpha}=D^{\alpha} D^{\alpha}$ and $D_{x}^{\alpha} u=d^{\alpha} u(x) / d x^{\alpha}$. 
The rest of the paper is organized as follows. In section 2 we list some basic definitions and properties of the fractional calculus theory. Adomian decomposition method is given in section 3. The numerical experiments are provided in section 4 and conclusion is in section 5 .

\section{Fractional derivative}

We give some basic definitions and properties of the fractional calculus theory which are used further in this paper.

Definition 2.1[25] Assume $f: \mathbb{R} \rightarrow \mathbb{R}, x \rightarrow f(x)$, denote a continuous (but not necessarily differentiable) function, and let the partition $h>0$ in the interval $[0,1]$. Through the fractional Riemann Liouville integral

$$
I_{x}^{\alpha} f(x)=\frac{1}{\Gamma(\alpha)} \int_{0}^{\mathrm{x}}(\mathrm{x}-\xi)^{\alpha-1} f(\xi) d \xi, \quad \alpha>0,
$$

the modified Riemann-Liouville derivative is defined as

$$
D_{x}^{\alpha} f(x)=\frac{1}{\Gamma(\mathrm{n}-\alpha)} \frac{\mathrm{d}^{\mathrm{n}}}{\mathrm{dx}^{\mathrm{n}}} \int_{0}^{\mathrm{x}}(\mathrm{x}-\xi)^{\mathrm{n}-\alpha}(f(\xi)-f(0)) d \xi,
$$

where $x \in[0,1], n-1 \leq \alpha<n$ and $n \geq 1$.

G. Jumarie's derivative is defined through the fractional difference [20]

$$
\Delta^{\alpha} f(x)=(F W-1)^{\alpha} f(x)=\sum_{k=0}^{\infty}(-1)^{k}\left(\begin{array}{l}
\alpha \\
k
\end{array}\right) f[x+(\alpha-k) h],
$$

where $F W f(x)=f(x+h)$. Then the fractional derivative is defined as the following limit,

$$
f^{\alpha}(x)=\lim _{h \rightarrow 0} \frac{\Delta^{\alpha} f(x)}{h^{\alpha}} .
$$

The proposed modified Riemann-Liouville derivative as shown in Eq.(2.2) is strictly equivalent to Eq.(2.4). Meanwhile, we would introduce some properties of the fractional modified Riemann-Liouville derivative in Eqs.(2.5) and (2.6).

(a) Fractional Leibniz product law

(b) Fractional Leibniz formulation

$$
D_{x}^{\alpha}(u v)=u^{(\alpha)} v+u v^{(\alpha)} .
$$

$$
I_{x}^{\alpha} D_{x}^{\alpha} f(x)=f(x)-f(0), \quad 0<\alpha \leq 1 .
$$

Therefore, the integration by part can be used during the fractional calculus

(c) Integration with respect to $(d x)^{\alpha}$.

$$
I_{b}^{\alpha}\left(u^{(\alpha)} v\right)=\left.(u v)\right|_{a} ^{b}-I_{b}^{\alpha}\left(u v^{(\alpha)}\right) .
$$

Assume $f(x)$ denote a continuous $\mathbb{R} \rightarrow \mathbb{R}$ function. We use the following equality for the integral w.r.t. $(d x)^{\alpha}$

(d)

$$
\begin{aligned}
I_{x}^{\alpha} f(x)= & \frac{1}{\Gamma(\alpha)} \int_{0}^{\mathrm{x}}(\mathrm{x}-\xi)^{\alpha-1} f(\xi) d \xi \\
& =\frac{1}{\Gamma(1+\alpha)} \int_{0}^{x} f(\xi)(d \xi)^{\alpha}, \quad 0<\alpha \leq 1 .
\end{aligned}
$$

$$
\begin{gathered}
D^{\alpha} x^{\gamma}=\Gamma(\gamma+1) \Gamma^{-1}(\gamma+1+\alpha) x^{\gamma-\alpha}, \quad \gamma>0, \\
{[f(u(x))]^{(\alpha)}=f_{u}^{\prime}(u) u^{(\alpha)}(x)} \\
=f_{u}^{(\alpha)}(u)\left(u_{x}^{\prime}\right)^{\alpha} .
\end{gathered}
$$

\section{Adomian decomposition method}

In this section, Adomian decomposition method is extended to fractional case in sense of modified Riemann-Liouville derivative.

Consider a fractional nonlinear differential equation in the form

$$
\begin{aligned}
& L^{\alpha}(y)-N(y)=f, \quad y=y(x), \\
& y^{(k \alpha)}(0)=c_{k}, k=0,1, \ldots, n-1,
\end{aligned}
$$


where $L^{\alpha}=\frac{d^{n \alpha}}{d x^{n \alpha}}=\underbrace{D^{\alpha} D^{\alpha} \ldots D^{\alpha}}_{n}$ is the fractional derivative of $n \alpha$-order, then the corresponding $L^{-\alpha}$ operator can be written in the form

$$
L^{-\alpha}(\cdot)=\frac{1}{\Gamma^{n}(1+\alpha)} \int_{0}^{x} \int_{0}^{t_{n}} \int_{0}^{t_{n-1}} \ldots \int_{0}^{t_{2}}(\cdot)\left(d t_{1}\right)^{\alpha} \ldots\left(d t_{n-1}\right)^{\alpha}\left(d t_{n}\right)^{\alpha} .
$$

$\frac{1}{\Gamma(1+\alpha)} \int_{0}^{x}(\cdot)\left(d t_{n}\right)^{\alpha}$ is the Riemann-Liouville integration.

The nonlinear term, $N(y)$, is expressed by an infinite series of the Adomian polynomials

$$
\begin{gathered}
N(y)=\sum_{n=0}^{\infty} A_{n}, \\
A_{n}\left(y_{0}, y_{1}, \ldots, y_{n}\right)=\left.\frac{1}{n !} \frac{d^{n}}{d \lambda^{n}}\left(N\left[\sum_{k=0}^{\infty} \lambda^{k} y_{k}\right]\right)\right|_{\lambda=0^{\prime}} \quad n \geq 0 .
\end{gathered}
$$

Using the Maclaurin series of fractional order [26] and applying the operator $L^{-\alpha}$ to both sides of Eq.(3.1), we have

$$
y(x)=\sum_{k=0}^{n-1} \frac{x^{k \alpha}}{\Gamma(1+k \alpha)} y^{(k \alpha)}(0)+L^{-\alpha} N(y)+L^{-\alpha} f, \quad 0<\alpha \leq 1 .
$$

The Adomian decomposition method suggests the solution be decomposed into the infinite series of components

where $N=\sum_{n=0}^{\infty} A_{n}$, and

$$
y(x)=\sum_{k=0}^{\infty} y_{k}(x),
$$

$$
A_{n}\left(y_{0}, y_{1}, \ldots, y_{n}\right)=\left.\frac{1}{n !} \frac{d^{n}}{d \lambda^{n}}\left(N\left[\sum_{k=0}^{\infty} \lambda^{k} y_{k}\right]\right)\right|_{\lambda=0^{\prime}} \quad n \geq 0 .
$$

The iterates are determined by following recursive way

and

$$
y_{0}(x)=\sum_{k=0}^{n-1} \frac{x^{k \alpha}}{\Gamma(1+k \alpha)} y^{(k \alpha)}(0)+L^{-\alpha} f
$$

Finally, we approximate the solution by the truncated series

$$
y_{k+1}(x)=L^{-\alpha} A_{n}(x), \quad n \geq 0 .
$$

$$
\Phi_{N}(x)=\sum_{n=0}^{N-1} y_{n}(x), \quad \lim _{N \rightarrow \infty} \Phi_{N}(x)=y(x) .
$$

\section{Examples}

In this section, we solve three examples by Adomian decomposition method.

Example 1. Consider fractional Bratu-type equation with initial condition

$$
\begin{gathered}
D_{x}^{2 \alpha} u(x)-2 e^{u(x)}=0, \quad 0<\alpha \leq 1, \quad 0<x<1, \\
u(0)=u^{(\alpha)}(0)=0 .
\end{gathered}
$$

The exact solution of Eq.(4.1) in $\alpha=1$ is

$$
u(x)=-2 \ln (\cos x) .
$$

Using the Maclaurin series of fractional order, we can determine the initial value or a trial function

The generalized iteration procedures can be given as

$$
u_{0}(x)=u(0)+\frac{u^{(\alpha)}(0)}{\Gamma(1+\alpha)} x^{\alpha}=0 .
$$

where $A_{k}$ is a Adomian polynomials

$$
u_{k+1}(x)=\frac{1}{\Gamma^{2}(1+\alpha)} \int_{0}^{x} \int_{0}^{t_{2}} A_{k}\left(d t_{1}\right)^{\alpha}\left(d t_{2}\right)^{\alpha}, \quad k \geq 0 .
$$

$$
\begin{gathered}
A_{0}=N\left(u_{0}\right) \Rightarrow A_{0}=2, \\
A_{1}=u_{1} N^{\prime}\left(u_{0}\right) \Rightarrow A_{0}=2 u_{1}, \\
A_{2}=u_{2} N^{\prime}\left(u_{0}\right)+\frac{1}{2}\left(u_{1}\right)^{2} N^{\prime \prime}\left(u_{0}\right) \Rightarrow A_{0}=2 u_{1}+\left(u_{1}\right)^{2},
\end{gathered}
$$


In this example, the nonlinear term is $N=2 e^{u}$.

By applied Eq.(3.8) and Eq.(4.3), we obtain

$$
\begin{aligned}
& u_{1}(x)=\frac{2}{\Gamma(1+2 \alpha)} x^{2 \alpha} \\
& u_{1}(x)=\frac{4}{\Gamma(1+4 \alpha)} x^{4 \alpha}
\end{aligned}
$$

Therefore

$$
u(x)=u_{0}+u_{1}+u_{2}+\cdots=\frac{2}{\Gamma(1+2 \alpha)} x^{2 \alpha}+\frac{4}{\Gamma(1+4 \alpha)} x^{4 \alpha}+\cdots .
$$

The exact solution for $\alpha=1$ and approximate solutions for $\alpha=0.5,0.6, \cdots, 1$ are shown in Fig.1.

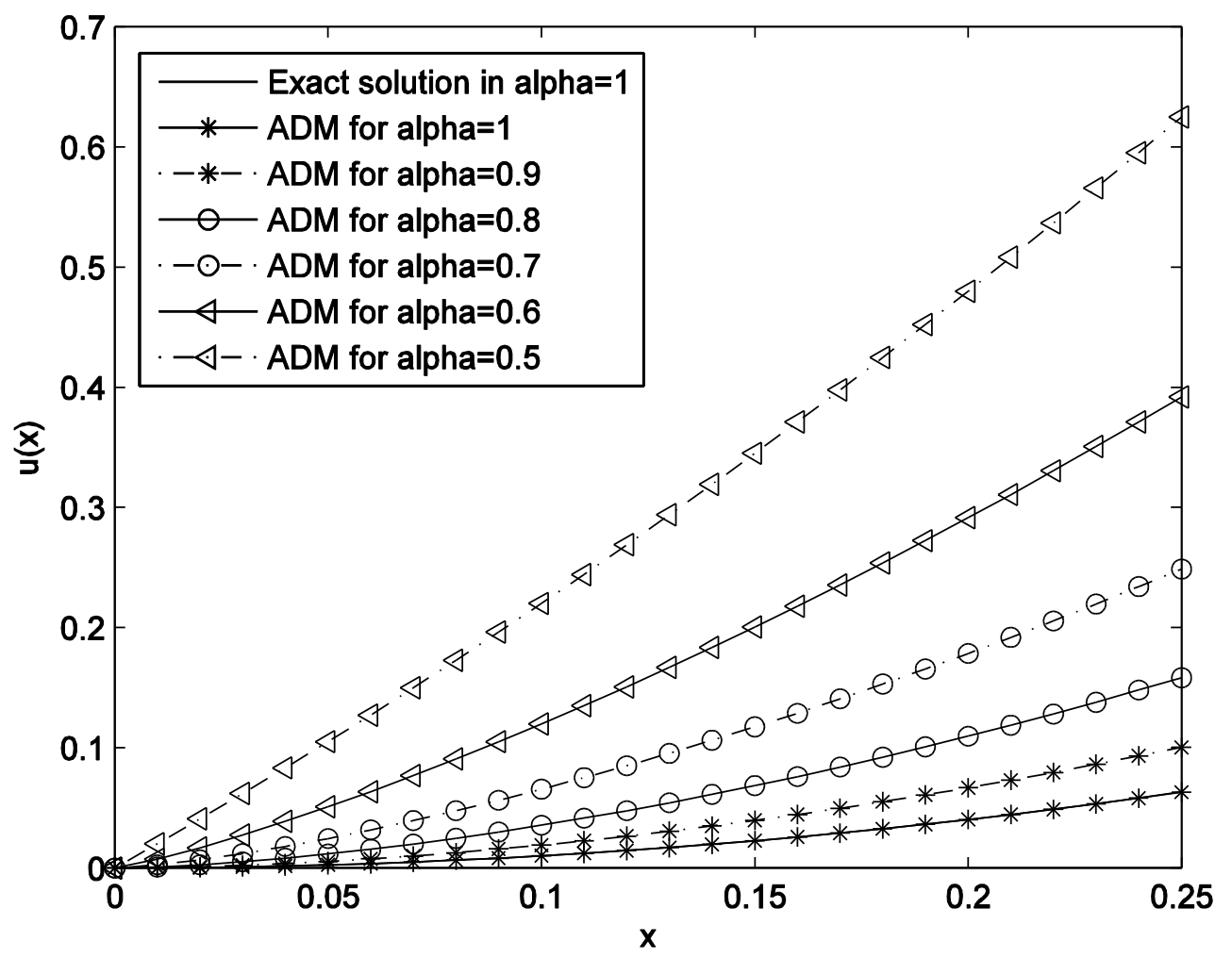

Figure 1: The exact solution in $\alpha=1$ and approximate solutions of ADM.

Example 2. Consider fractional Bratu-type equation with initial condition

$$
\begin{gathered}
D_{x}^{2 \alpha} u(x)-\pi^{2} e^{u(x)}=0, \quad 0<\alpha \leq 1, \quad 0<x<1, \\
u(0)=0, \quad u^{(\alpha)}(0)=\pi .
\end{gathered}
$$

The exact solution of Eq.(4.5) in $\alpha=1$ is

$$
u(x)=-\ln (1-\sin (\pi x)) \text {. }
$$

Using the Maclaurin series of fractional order, we can determine the initial value or a trial function

The generalized iteration procedures can be given as

$$
u_{0}(x)=u(0)+\frac{u^{(\alpha)}(0)}{\Gamma(1+\alpha)} x^{\alpha}=\frac{\pi x^{\alpha}}{\Gamma(1+\alpha)} .
$$




$$
u_{k+1}(x)=\frac{1}{\Gamma^{2}(1+\alpha)} \int_{0}^{x} \int_{0}^{t_{2}} A_{k}\left(d t_{1}\right)^{\alpha}\left(d t_{2}\right)^{\alpha}, \quad k \geq 0,
$$

where $A_{k}$ is a Adomian polynomials

$$
\begin{gathered}
A_{0}=N\left(u_{0}\right) \Rightarrow A_{0}=\pi^{2} e^{u_{0}} \\
A_{1}=u_{1} N^{\prime}\left(u_{0}\right) \Rightarrow A_{0}=\pi^{2} e^{u_{0}} u_{1}, \\
A_{2}=u_{2} N^{\prime}\left(u_{0}\right)+\frac{1}{2}\left(u_{1}\right)^{2} N^{\prime \prime}\left(u_{0}\right) \Rightarrow A_{0}=\pi^{2} e^{u_{0}}\left(u_{1}+\frac{1}{2}\left(u_{1}\right)^{2}\right),
\end{gathered}
$$

In this example, the nonlinear term is $N=\pi^{2} e^{u}$.

By applied Eq.(3.8) and Eq.(4.7), we obtain

$$
\begin{gathered}
u_{1}(x)=e^{\frac{\pi x^{\alpha}}{\Gamma(1+\alpha)}}+\frac{\pi x^{\alpha}}{\Gamma(1+\alpha)}-1, \\
u_{2}(x)=\frac{1}{4} e^{\frac{2 \pi x^{\alpha}}{\Gamma(1+\alpha)}}-\frac{\pi x^{\alpha}}{\Gamma(1+\alpha)} e^{\frac{\pi x^{\alpha}}{\Gamma(1+\alpha)}}+e^{\frac{\pi x^{\alpha}}{\Gamma(1+\alpha)}}-\frac{\pi x^{\alpha}}{2 \Gamma(1+\alpha)}-\frac{5}{4}
\end{gathered}
$$

Therefore

$$
\begin{gathered}
u(x)=u_{0}+u_{1}+u_{2}+\cdots=\frac{\pi x^{\alpha}}{\Gamma(1+\alpha)}+\left(e^{\frac{\pi x^{\alpha}}{\Gamma(1+\alpha)}}+\frac{\pi x^{\alpha}}{\Gamma(1+\alpha)}-1\right)+\left(\frac{1}{4} e^{\frac{2 \pi x^{\alpha}}{\Gamma(1+\alpha)}}-\frac{\pi x^{\alpha}}{\Gamma(1+\alpha)} e^{\frac{\pi x^{\alpha}}{\Gamma(1+\alpha)}}+e^{\frac{\pi x^{\alpha}}{\Gamma(1+\alpha)}}-\right. \\
\left.\frac{\pi x^{\alpha}}{2 \Gamma(1+\alpha)}-\frac{5}{4}\right)+\cdots
\end{gathered}
$$

The exact solution for $\alpha=1$ and approximate solutions for $\alpha=0.5,0.6, \cdots, 1$ are shown in Fig.2. 


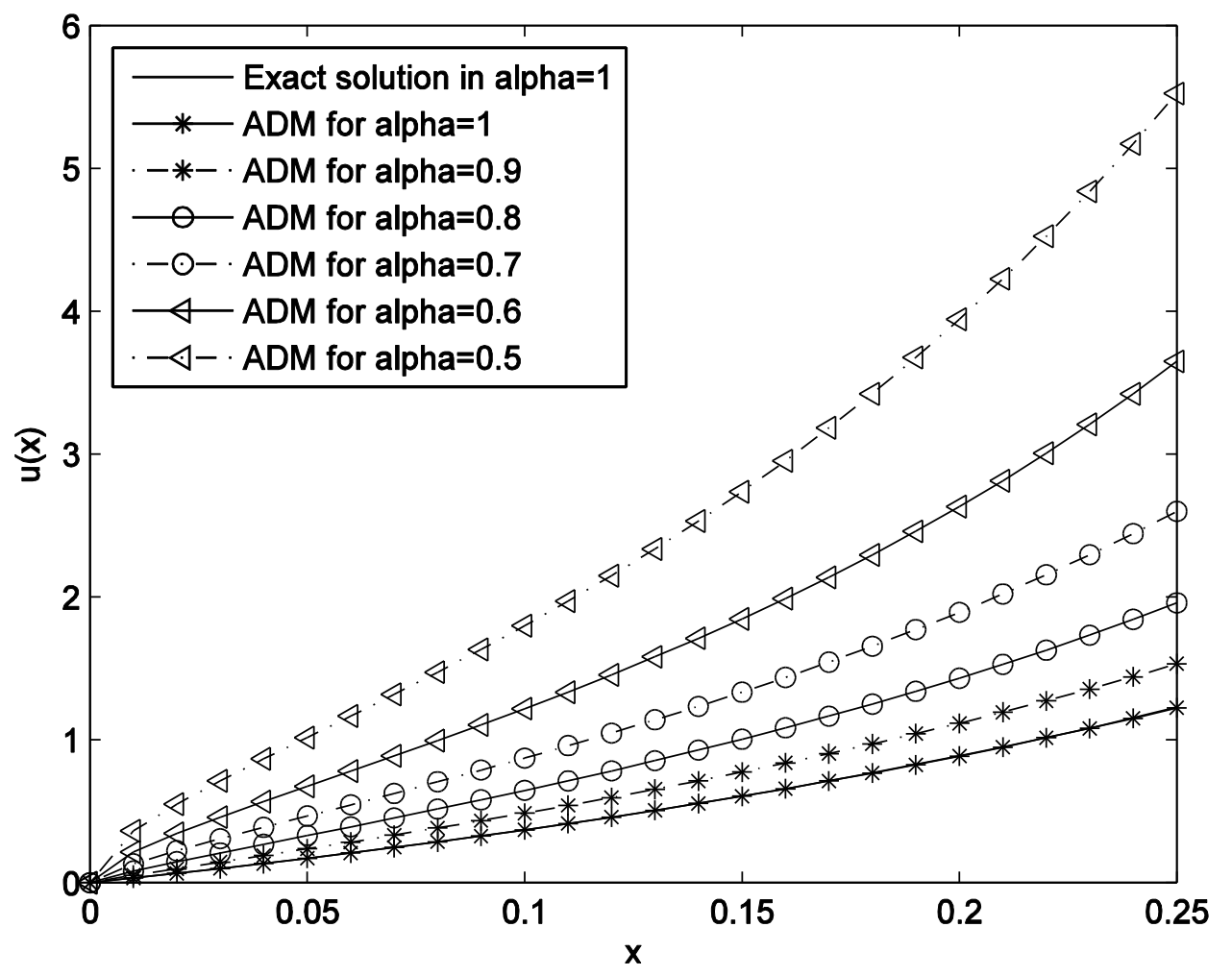

Figure 2: The exact solution in $\alpha=1$ and approximate solutions of ADM.

Example 3. Consider fractional Bratu-type equation with initial condition

$$
\begin{gathered}
D_{x}^{2 \alpha} u(x)+\pi^{2} e^{-u(x)}=0, \quad 0<\alpha \leq 1,0<x<1, \\
u(0)=0, \quad u^{(\alpha)}(0)=\pi .
\end{gathered}
$$

The exact solution of Eq.(4.5) in $\alpha=1$ is

$$
u(x)=\ln (1+\sin (\pi x)) .
$$

Using the Maclaurin series of fractional order, we can determine the initial value or a trial function

$$
u_{0}(x)=u(0)+\frac{u^{(\alpha)}(0)}{\Gamma(1+\alpha)} x^{\alpha}=\frac{\pi x^{\alpha}}{\Gamma(1+\alpha)} .
$$

The generalized iteration procedures can be given as

where $A_{k}$ is a Adomian polynomials

$$
u_{k+1}(x)=\frac{1}{\Gamma^{2}(1+\alpha)} \int_{0}^{x} \int_{0}^{t_{2}} A_{k}\left(d t_{1}\right)^{\alpha}\left(d t_{2}\right)^{\alpha}, \quad k \geq 0,
$$

$$
\begin{gathered}
A_{0}=N\left(u_{0}\right) \Rightarrow A_{0}=-\pi^{2} e^{-u_{0}} \\
A_{1}=u_{1} N^{\prime}\left(u_{0}\right) \Rightarrow A_{0}=\pi^{2} e^{-u_{0}} u_{1} \\
A_{2}=u_{2} N^{\prime}\left(u_{0}\right)+\frac{1}{2}\left(u_{1}\right)^{2} N^{\prime \prime}\left(u_{0}\right) \Rightarrow A_{0}=\pi^{2} e^{-u_{0}}\left(u_{1}-\frac{1}{2}\left(u_{1}\right)^{2}\right)
\end{gathered}
$$

In this example, the nonlinear term is $N=-\pi^{2} e^{-u}$.

By applied Eq.(3.8) and Eq.(4.11), we obtain

$$
u_{1}(x)=-e^{\frac{-\pi x^{\alpha}}{\Gamma(1+\alpha)}}-\frac{\pi x^{\alpha}}{\Gamma(1+\alpha)}+1,
$$




$$
u_{2}(x)=-\frac{1}{4} e^{\frac{-2 \pi x^{\alpha}}{\Gamma(1+\alpha)}}-\frac{\pi x^{\alpha}}{\Gamma(1+\alpha)} e^{\frac{-\pi x^{\alpha}}{\Gamma(1+\alpha)}}-e^{\frac{-\pi x^{\alpha}}{\Gamma(1+\alpha)}}-\frac{\pi x^{\alpha}}{2 \Gamma(1+\alpha)}+\frac{5}{4}
$$

Therefore

$$
\begin{gathered}
u(x)=u_{0}+u_{1}+u_{2}+\cdots=\frac{\pi x^{\alpha}}{\Gamma(1+\alpha)}+\left(-e^{\frac{-\pi x^{\alpha}}{\Gamma(1+\alpha)}}-\frac{\pi x^{\alpha}}{\Gamma(1+\alpha)}+1\right)+\left(-\frac{1}{4} e^{\frac{-2 \pi x^{\alpha}}{\Gamma(1+\alpha)}}-\frac{\pi x^{\alpha}}{\Gamma(1+\alpha)} e^{\frac{-\pi x^{\alpha}}{\Gamma(1+\alpha)}}-\right. \\
\left.e^{\frac{-\pi x^{\alpha}}{\Gamma(1+\alpha)}}-\frac{\pi x^{\alpha}}{2 \Gamma(1+\alpha)}+\frac{5}{4}\right)+\cdots .
\end{gathered}
$$

The exact solution for $\alpha=1$ and approximate solutions for $\alpha=0.5,0.6, \cdots, 1$ are shown in Fig.3.

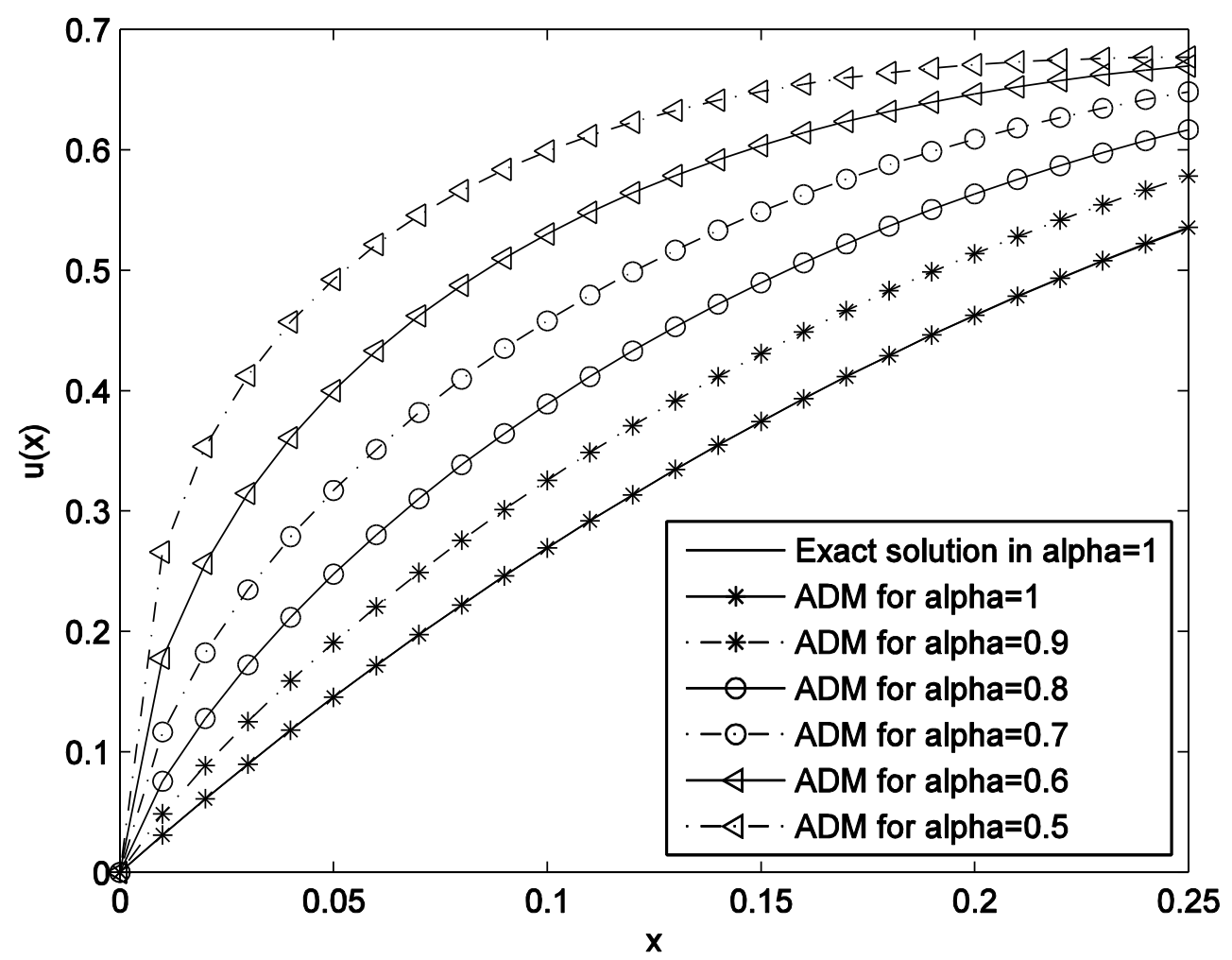

Figure 3: The exact solution in $\alpha=1$ and approximate solutions of ADM.

\section{Conclusion}


The Adomian decomposition method for fractional differential equations has been extensively worked out for many years. In this study, the approximate solution of a fractional Bratu-type equations are investigated by Adomian decomposition method. The results show that the Adomian decomposition method is effective and very simple.

\section{References}

1. F.B.M. Duarte, J.A. Tenreiro Machado, Chaotic phenomena and fractional-order dynamics in the trajectory control of redundant manipulators, Nonlinear Dyn., 29 (2002) 342-362.

2. O.P. Agrawal, A general formulation and solution scheme for fractional optimal control problems, Nonlinear Dyn., 38 (2004) 323-337.

3. N. Engheta, On fractional calculus and fractional multipoles in electromagnetism, IEEE T. Antenn. Propag., 44 (1996) 554-566.

4. R.L. Magin, Fractional calculus models of complex dynamics in biological tissues, Comput. Math. Appl., 59 (2010) 1586-1593.

5. V.V. Kulish, Jos L. Lage, Application of fractional calculus to fluid mechanics, J. Fluids Eng., 124 (2002), doi:10.1115/1.1478062.

6. K.B. Oldham, Fractional differential equations in electrochemistry, Adv. Eng. Soft., 41 (2010) 9-12.

7. V. Gafiychuk, B. Datsko, V. Meleshko, Mathematical modeling of time fractional reaction diffusion systems, J. Comput. Appl. Math., 220 (2008) 215-225.

8. C. Lederman, J-M Roquejoffre, N. Wolanski, Mathematical justification of a nonlinear integrodifferential equation for the propagation of spherical flames, Ann. di Mate., 183 (2004) 173-239. 9. F. Mainardi, Fractional calculus: some basic problems in continuum and statistical mechanics, in: A. Carpinteri, F. Mainardi (Eds.), Fractals and Fractional Calculus in Continuum Mechanics, SpringerVerlag, New York, (1997), pp. 291-348.

10. F.C. Meral, T.J. Royston, R. Magin, Fractional calculus in viscoelasticity: an experimental study, Commun. Nonl. Sci. Num. Sim., 15 (2010) 939-945.

11. H. Jafari, S. Seifi, Homotopy Analysis Method for solving linear and nonlinear fractional diffusionwave equation, Commun. Nonli. Science Numer. Simul., 14 ( 5) (2009) 2006--2012.

12. V. Daftardar-Gejji, H. Jafari, Solving a multi-order fractional differential equation using adomian decomposition, Appl. Math. Comput., 189 (2007) 541-548.

13. O. Abdulaziz, I. Hashim, S. Momani, Solving systems of fractional differential equations by homotopy-perturbation method, Phys. Lett., A 372 (2008) 451-459.

14. B. Ghazanfari, A.G. Ghazanfari, M. Fuladvand, Modification of the Homotopy Perturbation Method for Numerical Solution of Nonlinear Wave and System of Nonlinear Wave Equations, J. Math. Computer Sci. 3 (2011) 212-224.

15. M. Mahmoudi, M.V. Kazemi, Solving Singular BVPs Ordinary Differential Equations by Modified Homotopy Perturbation Method, J. Math. Computer Sci. 7 (2013) 138-143.

16. M. Rabbani, New Homotopy Perturbation Method to Solve Non-Linear Problems, J. Math. Computer Sci. 7 (2013) 272-275.

17. I. Hashim, O. Abdulaziz, S. Momani, Homotopy analysis method for fractional IVPs, Commun. Nonlinear Sci. Numer. Simul., 14 (2009) 674-684.

18. G. Wu, E.W.M. Lee, Fractional variational iteration method and its application, Phys. Lett., A 374 (2010) 2506-2509. 
19. Z. Odibat, S. Momani, V. Suat Erturk, Generalized differential transform method: application to differential equations of fractional order, Appl. Math. Comput., 197 (2008) 467-477.

20. Y. Zhang, A finite difference method for fractional partial differential equation, Appl. Math. Comput., 215 (2009) 524-529.

21. A. Neamaty, B. Agheli, R. Darzi, Solving Fractional Partial Differential Equation by Using Wavelet Operational Method, J. Math. Computer Sci. 7 (2013) 230-240.

22. H. Azizi, Gh. Barid Loghmani A numerical method for space fractional diffusion equations using a semi-disrete scheme and Chebyshev collocation method, J. Math. Computer Sci. 8 (2014) 226-235. 23. G. Adomian, Solving Frontier Problems of Physics: The Decomposition Method, Kluwer Academic Publishers, Boston, (1994).

24. G. Adomian, A review of the decomposition method in applied mathematics, J. Math. Anal. Appl., 135 (1988) 501-544.

25. G. Jumarie, Modified Riemann-Liouville derivative and fractional Taylor series of non-differentiable functions further results, Comput. Math. Appl., 51 (2006) 1367-1376.

26. Y. Chen, Y. Yan, K.W. Zhang, On the local fractional derivative, J. Math. Anal. Appl., 362 (2010) $17-33$. 